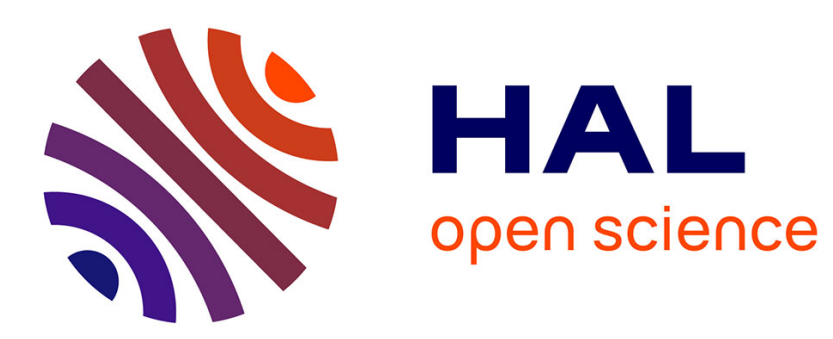

\title{
Légitimer et disqualifier: les fake news saisies comme opportunité de normalisation du champ journalistique
}

\author{
Marie-Noëlle Doutreix, Lionel Barbe
}

\section{To cite this version:}

Marie-Noëlle Doutreix, Lionel Barbe. Légitimer et disqualifier: les fake news saisies comme opportunité de normalisation du champ journalistique. Études de communication - Langages, information, médiations, 2019, Fake-News! Pouvoirs et conflits autour de l'énonciation publique du " vrai ", 53, pp.49-66. 10.4000/edc.9242 . hal-03083921

\section{HAL Id: hal-03083921 \\ https://hal.science/hal-03083921}

Submitted on 19 Dec 2020

HAL is a multi-disciplinary open access archive for the deposit and dissemination of scientific research documents, whether they are published or not. The documents may come from teaching and research institutions in France or abroad, or from public or private research centers.
L'archive ouverte pluridisciplinaire HAL, est destinée au dépôt et à la diffusion de documents scientifiques de niveau recherche, publiés ou non, émanant des établissements d'enseignement et de recherche français ou étrangers, des laboratoires publics ou privés. 


\title{
Légitimer et disqualifier : les fake news saisies comme opportunité de normalisation du champ journalistique
}

\author{
Marie-Noëlle Doutreix \\ Université de Lorraine CREM / Université Paris 3 CIM \\ mn.doutreix@gmail.com \\ Lionel Barbe \\ Université de Nanterre DICEN-IDF \\ lionel.barbe@parisnanterre.fr
}

Cet article s'intéresse à la manière dont l'actualité autour des fake news est saisie par une diversité d'acteurs traditionnels du champ médiatique (ici Le Monde, l'Agence France Presse et Reporters Sans Frontières) pour revaloriser les pratiques professionnelles à l'aide d'outils normatifs qui identifieraient les médias fiables. La médiatisation des fake news comme phénomène massif et disruptif leur permet de proposer un réagencement du champ journalistique. En effet, dans un contexte concurrentiel difficile, les divers partenariats entrepris autour des fake news, avec Google et Facebook principalement, leur permet de se réattribuer une place centrale dans la production et la diffusion de l'information. Nous montrerons que cette reconfiguration s'opère aussi à travers une disqualification des littéracies informationnelles des internautes dont la participation est tantôt sollicitée, tantôt décriée, en fonction des intérêts en présence. 
Notre recherche part du constat que certains médias traditionnels ${ }^{1}$ présentent les fake news comme un problème lié à Internet et en particulier à la circulation de l'information sur les réseaux sociaux numériques à laquelle participeraient les publics. Les internautes, s'ils ne sont pas producteurs de fake news, n'en seraient pas moins les acteurs de leur propagation. Comme nous l'observerons, ce parti pris s'avère guidé par des enjeux de reconnaissance et de place dans le champ de l'information. Les réponses que ces médias traditionnels proposent aux fake news - qu'ils contribuent eux-mêmes largement à faire émerger comme problème public - relèvent d'une conception verticale de la fiabilité de l'information et de dispositifs « top-down » classiques. Nous étudierons deux dispositifs représentatifs de cette manière de se saisir des fake news dans une optique de (re)légitimation et de reprise en main de l'information par les médias traditionnels dans un contexte de défiance à leur égard : le Décodex lancé en 2017 et le projet Journalism Trust Initiative officialisé en 2018 et actuellement en cours de définition. Les deux dispositifs ont en commun de vouloir renseigner le public sur la fiabilité des médias en ligne.

Nous montrerons, dans un premier temps, comment l'accent mis sur les fake news renouvelle les discours de disqualification des publics en analysant les discours d'accompagnement du lancement du Décodex par le journal Le Monde. Puis nous étudierons la " réponse » qu'apporte le réseau de grands médias regroupés à travers Journalism Trust Initiative. Ce projet promeut la mise en place d'une norme internationale de production de contenus journalistiques et d'un système de confiance par labellisation ou certification. Nous mettrons en exergue que cette initiative reproduit, plutôt qu'elle ne résout, la fracture entre les médias prescripteurs de ce qui doit être considéré comme fiable et les lecteurs qui seraient en incapacité de discerner la fiabilité d'une source. Enfin, nous montrerons comment ces discours de disqualification, plus ou moins sous-tendus ou affichés, peuvent aussi évoluer en fonction des intérêts des acteurs en place, notamment face à l'irruption ou à l'ascension de nouveaux acteurs. Le cas de Wikipédia illustrera ainsi les déplacements des discours de disqualification tout en montrant qu'un processus davantage ouvert, horizontal et décentralisé peut finalement être à même à la fois de gérer les fausses informations et de permettre la construction active par le lecteur/acteur d'une évaluation pertinente des contenus. On constatera que Wikipédia, initialement considérée comme peu fiable, a été convertie en outil majeur contre les fake news dans le cadre de son usage par Google - spécialement dans le cas de YouTube - et Facebook. Afin de traiter au mieux ces questions, nous procéderons par l'analyse qualitative de discours et par la mise en perspective socio-économique des projets et acteurs étudiés.

\section{Les discours sur les fake news, une réitération de la disqualification des publics dans la circulation de l'information : le cas du Décodex}

Les discours sur les fake news renvoient à l'antagonisme, réitéré sous de multiples formes dans la vie politique, entre savants et ignorants. Les savants, qui s'inquiètent de l'influence des fake news sur le comportement des individus, sauraient distinguer le vrai du faux ou tout au moins faire preuve d'esprit critique, tandis que les ignorants en seraient peu pourvus, croiraient les informations mensongères et agiraient sous leur influence. Ces types de discours relèvent de ce que Sandra Laugier et Albert Ogien (2017) ont qualifié d'antidémocratie, où ceux qui gouvernent dénient à ceux qui sont gouvernés la capacité de prendre des décisions qui les concernent. Nous souhaitons identifier la manière dont cette distinction se retrouve dans le champ de l'information sous diverses formes.

\footnotetext{
${ }^{1}$ Terme générique que nous emploierons pour désigner ici les journaux quotidiens français imprimés d'actualité de dimension nationale ou internationale et à grand tirage qui préexistaient au développement des pure players d'information.
} 
Le Décodex, créé à destination des internautes par l'équipe des Décodeurs du journal Le Monde, a été lancé en janvier 2017, en pleine période de prolifération des discours de mise en garde contre les fake news. Les discours présentant et entourant le Décodex sur sa page Internet participent de manière symptomatique au discrédit du rôle de l'internaute dans la circulation de l'information. Les propos du Décodex se démarquent en effet par une condescendance envers le lecteur-diffuseur d'informations. Le premier sujet proposé à l'utilisateur sur la page du moteur de recherche interne du Décodex s'intitule « Pourquoi il est important de vérifier une information avant de la partager ». La responsabilité des usagers de l'information dans la circulation des fake news y est directement pointée, notamment avec l'intertitre "Responsables de ce que nous propageons » qui, bien qu'utilisant la première personne du pluriel, n'inclut en rien les pratiques journalistiques du Monde.

Cette distinction entre les professionnels du Monde et les internautes profanes s'explicite dès le troisième paragraphe, commençant par la phrase suivante «C'est pour vous aider dans cette démarche que nous avons développé toute une série d'outils et de guides pratiques ». Cette distinction se renforce encore dans la suite du paragraphe "L'idée n'étant pas d'imposer les contraintes des journalistes à tous les internautes (...)» qui souligne le sérieux des journalistes et l'écart nécessaire entre leurs pratiques et celles que pourrait acquérir tout un chacun. Le " nous " inclusif est évacué dans la mini bande dessinée concluant le texte où est mis en scène un internaute en colère, que l'on comprend indigné par une information qui en réalité serait fausse. La bande dessinée utilise l'interpellation par le " vous » et des injonctions sous les titres «attention!" et "Bon réflexe ! » clarifiant les bonnes et les mauvaises pratiques. Il ressort ainsi qu'à travers le Décodex, Le Monde se redessine une position de savoir, d'autorité et de garant des bonnes pratiques informationnelles. Conjointement à cette position de savoir, le service proposé par le Décodex repose sur l'idée que les publics n'ont pas les capacités par eux-mêmes de juger de la fiabilité d'un site internet.

Dans les propos du Décodex, le profane n'est pas seulement décrié pour sa contribution à l'encyclopédisme en ligne ou au journalisme participatif - comme ça a pu être le cas concernant Rue89 ou Agoravox- mais pour sa simple activité de transmission. Cette tension tout à la fois rappelle la disqualification de la foule, source de propagation de rumeurs, et met en exergue que, par la diffusion d'informations à grande échelle, les internautes prennent part (Rancière, 2004b) à un processus auquel, visiblement, ils ne sont pas pleinement autorisés.

Pourtant, les usagers des réseaux sociaux numériques, et en particulier de Twitter, partagent aussi pour beaucoup des informations provenant de grands médias, issus de l'imprimé ou télévisuels. Et certaines des fausses informations qui circulent sur Twitter proviennent de ces médias-ci (Alloing, Vanderbiest, 2018). Certes, ces informations ne sont pas intentionnellement fausses, mais « Cette distinction fondée sur l'intentionnalité, souvent difficile à déterminer, de la personne qui produit l'information, permet surtout d'immuniser par avance le journalisme professionnel qui pourrait ainsi être à l'origine de mauvais articles, mais jamais ou très rarement de "fake news" » (Michel, 2017).

Symétriquement au processus de valorisation du journalisme traditionnel dans l'espace public (Bigot, 2017), la disqualification de l'internaute en situation de partage d'informations s'avère problématique parce qu'elle va dans le sens d'une mise en cause de la capacité du citoyen à participer aux débats publics. Si cette exclusion du lecteur de la réflexion sur la qualité de l'information est ancienne (Ouakrat, 2019), elle se réitère dans le cadre du projet journalistique contre la désinformation et les fake news intitulé Journalism Trust Initiative. 


\section{Journalism Trust Initiative, la certification comme outil de distinction et de récompense des bonnes pratiques informationnelles}

Nous faisons l'hypothèse que la mise en exergue du problème des fausses informations par la dénomination "fake news» - et par le vocable qui leur est rattaché «prolifération », « explosion », «propagation» - a été saisie par certains acteurs traditionnels du champ journalistique pour tenter de retrouver une place centrale dans la diffusion de l'information. Il semble que les fake news soient invoquées pour revaloriser les pratiques professionnelles, même si le terme est parfois décrié par ceux-là mêmes qui le mobilisent ${ }^{2}$. L'idée généralement portée par les journalistes serait que si le faux peut aussi être du côté des médias, le faux intentionnel associé au concept de fake news se situerait du côté des personnalités politiques et des internautes (Sauvé, 2019).

Comme nous l'avons décrit avec le cas du Décodex, la partition entre médias de référence et pratiques des internautes se double de celle entre les médias dignes de confiance et ceux qui ne seraient pas fiables. C'est cette dernière distinction que propose de formaliser Journalism Trust Initiative en élaborant un modèle de certification des acteurs de l'information. Ce projet, à l'initiative de Reporters sans frontières et pensé à l'échelle européenne, rassemble des acteurs divers : journalistes mais aussi annonceurs, organismes de normalisation, spécialistes des plateformes et des algorithmes ou encore associations de professionnels et d'industriels des secteurs des médias, de l'internet ou de la publicité. La certification reposerait sur le respect de quatre standards jouant le rôle d'indicateurs : la transparence des médias, l'indépendance éditoriale, la mise en œuvre de méthodes journalistiques et le respect des règles déontologiques ${ }^{3}$. Les règles et critères précis sont en cours d'élaboration et seraient présentés fin 2019. Ils devraient posséder comme caractéristique commune que leur application effective puisse être vérifiée. Notons que ceuxci ne porteront pas sur la qualité finale des contenus mais sur les procédés de production de l'information ${ }^{4}$.

Le développement des fake news et de la désinformation sur Internet est présenté comme le point de départ de l'initiative. Le terme est employé dès le premier paragraphe du document Approved Project Plan, qui constitue la feuille de route de Journalism Trust Initiative :

In our digital age, it has become increasingly difficult to distinguish information shaped by vested interests from that produced by independent and fair news professionals. An open and honest public debate, aimed at informing the general public, is more and more difficult to sustain, especially when propaganda and "fake news" seem on the rise and trust in institutions and the media is declining. ${ }^{5}$

Le projet s'inscrit ainsi dans les initiatives actuelles contre les fake news auxquelles il propose de fournir un cadre favorisant leur coordination et leur synchronisation. Journalism Trust Initiative déclare vouloir s'attaquer aux causes des fake news, en intervenant avant la publication de celles-ci et non en les rectifiant a posteriori ${ }^{6}$.

\footnotetext{
${ }^{2}$ Comité européen de normalisation, « Project Plan for the CEN Workshop on Journalism Trust Indicators », 29 mars 2018 , p. 11.

${ }^{3}$ Éric Scherer « Contre la désinformation, Reporters sans frontières lance la Journalism Trust Initiative », MétaMedia, 3 avril 2018.

4 «L'exercice ne porte donc pas sur la qualité des contenus en eux-mêmes, mais les méthodes conduisant à les obtenir. » https://normalisation.afnor.org/actualites/journalism-trust-initiative-afnor-compagne-rsf-partenaires/

${ }^{5}$ Comité européen de normalisation, « Project Plan for the CEN Workshop on Journalism Trust Indicators », 29 mars 2018.

${ }^{6}$ « Apporter des solutions face à la crise de confiance que connaît la presse, dont la légitimité à informer subit aujourd'hui la concurrence d'Internet, avec des méthodes conduisant parfois à biaiser l'information : voilà un
} 
S'il se présente comme visant à encourager la rigueur des pratiques professionnelles, quatre autres finalités se dessinent par une lecture attentive du projet :

- Se démarquer d'une partie de la concurrence amenée par Internet en augmentant la visibilité des membres du projet au sein des algorithmes, des réseaux sociaux numériques et d'autres plateformes numériques

- Obtenir davantage de revenus publicitaires

- Demander éventuellement des subventions publiques supplémentaires qui seraient attribuées aux médias certifiés

- Renforcer leur légitimité auprès du public en valorisant les normes professionnelles distinguant les médias vertueux des autres producteurs d'information.

De l'idée qu'il faut regagner la confiance du public arrive rapidement celle qu'il faut désormais récompenser les médias qui respectent les standards journalistiques. L'idée est de donner « des avantages concrets ${ }^{7}$ à ceux qui les mettent en œuvre, sachant que les standards de référence seront ceux élaborés par le consortium réuni au sein de Journalism Trust Initiative. La volonté de labelliser est liée à des enjeux économiques. D'une part, attirer les annonceurs qui veulent associer leur marque à des médias "propres" et espèrent ainsi obtenir une «liste blanche» de médias dans lesquels investir de la publicité sans prise de risque $^{8}$. D'autre part, demander à ce que, à terme, des subventions publiques d'aide aux médias se fondent sur ce label.

Enfin, une autre motivation semble résider dans la volonté de prévenir par l'autorégulation les régulations que les pouvoirs publics se montrent tentés d'imposer par la loi. Il s'agit alors pour les médias de faire la preuve de leur capacité à s'auto-organiser en proposant des solutions à la circulation de fausses informations. Alors que le recours au législatif constitue une voie envisagée par le gouvernement français, risquant ainsi de contraindre les médias, Journalism Trust Initiative transforme les fake news en une opportunité pour ces derniers. Notons que plusieurs des membres du projet ont déjà reconfiguré leur activité (ou les discours autour de leur activité) comme constituant une réponse aux fake news. Ainsi, l'AFP, membre central de Journalism Trust Initiative, a redessiné ces dernières années ses missions autour de la lutte contre les fake news, revendiquant de placer «le combat contre la prolifération des manipulations et fausses informations au cœur de sa mission $»{ }^{9}$. Pourtant, la question de la vérité, si elle est posée par le fact-checking à propos des informations à vérifier, n'est finalement pas centrale dans les discours de Journalism Trust Initiative sur les fake news. La garantie qu'est censée apporter la certification ne repose pas sur la vérité des contenus mais sur la fiabilité du processus de production prenant en compte la transparence et l'indépendance journalistiques.

Alors même que Journalism Trust Initiative dit vouloir s'appliquer à divers types de producteurs d'information, y compris les blogs, ce projet valide les acteurs en position de force au détriment des acteurs plus modestes (Ouakrat, 2019). De surcroît, les certifications présentent souvent un coût élevé pour ceux qui souhaitent en être membres, prix que ne pourront payer les médias les plus isolés, parmi lesquels nombre de médias indépendants que le label est justement censé aider et soutenir. En conséquence, ce qui pourrait jouer le rôle de devise du projet «Au lieu de combattre le mal, soutenir le bien ${ }^{10}$ pourrait aussi devenir

des enjeux partagé par Reporters sans frontières (RSF) en 2017 auprès d'AFNOR, l'organisme de normalisation français. » https://normalisation.afnor.org/actualites/journalism-trust-initiative-afnor-compagne-rsf-partenaires/

${ }^{7}$ Site internet de Reporters Sans Frontières https://rsf.org/fr/actualites/rsf-et-ses-partenaires-devoilent-lajournalism-trust-initiative-jti-un-dispositif-innovant-contre-la

${ }^{8}$ Éric Scherer « Contre la désinformation, Reporters sans frontières lance la Journalism Trust Initiative », MétaMedia, 3 avril 2018.

${ }^{9}$ https://factuel.afp.com/propos

10 "Instead of fighting the bad and trying to disclose lies on the Internet, this initiative will support the good and promote journalism that deserves the trust of its audience. », Comité européen de normalisation, «Project Plan for the CEN Workshop on Journalism Trust Indicators », 29 mars 2018, p. 13. 
«soutenir quelques-uns, même au détriment des autres ». En effet, si les médias certifiés montent en visibilité dans les algorithmes comme le projet le souhaite, et comme Google et Facebook semblent s'y engager, cela n'aura pas seulement l'effet de rendre invisibles les producteurs de fake news mais aussi tous les médias qui, pour des raisons diverses, ne bénéficieront pas de la certification.

\section{Les fake news comme levier de négociation avec les plateformes numériques}

Après les nombreux différends ayant divisé les médias traditionnels et Google, ceuxci semblent désormais trouver dans les fake news un intérêt commun. Les Décodeurs ont ainsi réussi à obtenir un partenariat privilégié avec le moteur de recherche et également avec le réseau social Facebook (Badouard, 2019). Concernant Journalism Trust Initiative, la question des algorithmes s'avère consubstantielle au projet au-delà de la répartition de la visibilité entre les médias. En effet, les actions de Google visent, d'une manière ou d'une autre, à nourrir le développement de son intelligence artificielle. Journalism Trust Initiative a non seulement conscience de ce type de contrepartie mais semble même vouloir y contribuer ${ }^{11}$. Or ce type de partenariat ne semble pas constituer une solution transparente et inclusive vis-à-vis des internautes.

L'irruption de la question des fake news dans le débat public constitue pour Google également une opportunité. En effet, le moteur de recherche est accusé depuis plusieurs années d'une baisse de qualité des résultats de recherche, soit en faveur de sites commerciaux, soit parce que la viralité des réseaux fait souvent remonter les résultats les plus sensationnalistes concernant l'actualité ${ }^{12}$ et donc les fake news. Google a significativement investi, à grand renfort d'annonces, pour lutter contre le phénomène ${ }^{13}$. Mais le moteur de recherche s'était contenté, jusqu'en 2017, d'améliorer ses algorithmes, sans grand résultat. Les partenariats avec des médias de référence comme Le Monde ou Journalism Trust Initiative lui permettent d'intégrer un système basé sur les labels, qui rompt avec son fonctionnement traditionnel, sans assumer directement la responsabilité du choix des informations à favoriser. Cette mise en avant de certains médias en raison de leur activité de fact-checking permettrait de rétablir un meilleur contrôle du comportement des internautes dans une logique de silos informationnels toujours plus rémunérateurs en matière de publicité ciblée. Ce renforcement «qualitatif », dont la conséquence première serait la réduction de la diversité des sources médiatiques accessibles dans les premiers résultats, permettrait également à Google, ou à Facebook, une meilleure maîtrise de leur image vis-à-vis des pouvoirs publics.

Pour comprendre les dynamiques en cours en France, il faut également prendre en compte le contexte socio-économique dans lequel évoluent aujourd'hui les médias traditionnels issus de l'imprimé. Confrontés historiquement à une longue érosion des tirages papiers, ceux-ci ont vu la crise s'accélérer avec Internet, victimes jusqu'au début des années 2000 d'un effet ciseau : recul constant des revenus du papier d'un côté, incapacité à monétiser correctement une audience internet en forte croissance de l'autre. Dans un contexte concurrentiel fort, le virage forcé vers le numérique a aussi mis les rédactions sous pression et considérablement modifié le rythme et les formats de traitement de l'information. Pour

\footnotetext{
11 "Furthermore, the interoperability of these standards with algorithms of search engines and social media should be considered, both strategically and technically. This also includes the incorporation of existing work in this field as well as new areas to develop, particularly looking at emerging artificial intelligence (AI) mechanisms, machine-learning, and automated content sourcing/production/distribution/delivery and engagement.", Comité européen de normalisation, « Project Plan for the CEN Workshop on Journalism Trust Indicators », 29 mars 2018, p. 13.

${ }_{12} \mathrm{https}$ ://www.silicon.fr/fake-news-lue-met-la-pression-sur-google-facebook-et-twitter-239313.html

13 https://www.huffingtonpost.fr/2018/03/20/google-va-investir-300-millions-de-dollars-pour-lutter-contre-lesfakes-news_a 23390915/
} 
survivre et maximiser le taux de clics, la tentation du sensationnalisme est grande. Les médias issus de l'imprimé ont dû s'adapter à ce nouveau contexte, encouragés par les groupes financiers entrés massivement dans leurs capitaux au cours de la dernière décennie. En France, Libération a par exemple vu ses tirages papier baisser de moitié en dix ans seulement, ne vendant plus en 2018 (diffusion payée), que 67000 exemplaires en moyenne ${ }^{14}$, en baisse de $10 \%$ sur seulement un an. Pour tous les médias traditionnels, la transition numérique est en cours avec des revenus papier qui s'effondrent mais des revenus numériques en forte croissance pour ceux qui savent s'adapter comme Le Monde, avec par exemple une hausse des abonnements Internet de $44 \%$ en $2017^{15}$.

À ce contexte défavorable s'ajoute une crise de confiance généralisée vis-à-vis de la presse considérée par beaucoup d'internautes comme étant désormais à la solde des grands groupes et des politiques ${ }^{16}$. Même si les médias alternatifs ne bénéficient pas toujours d'une meilleure réputation, pour d'autres motifs, la défiance vis-à-vis de ce qui est considéré comme institutionnel leur a permis de récupérer une audience souhaitant un plus grand pluralisme dans l'information.

\section{Déplacements et reconfigurations des discours de disqualification}

Nos recherches tendent à observer que les discours de disqualification ou de valorisation se déplacent en fonction des partenariats réalisés entre les acteurs de l'information numérique. Avant que la question des internautes propagateurs de fake news sur les réseaux sociaux ne s'installe sur le devant de la scène, celle des internautes rédacteurs d'informations fausses ou approximatives sur Wikipédia a fortement occupé l'arène médiatique. Les discours actuels sur les fake news relèvent d'une même position normative que les critiques qui ont accompagné les débuts de Wikipédia, qualifiée de "soupe d'opinions » et de « fast-science $»{ }^{17}$. Peut-on pour autant effectuer des parallèles entre les critiques initiales, et parfois persistantes, envers Wikipédia, et celles, plus actuelles, touchant les fake news? Ces diverses critiques se rejoignent en cela qu'elles remettent en cause plus globalement les internautes dans leur capacité de discernement.

Concernant Wikipédia, Alexandre Moatti montre comment certains milieux intellectuels, dans une posture assimilable à celle de "gardiens du temple », ont essayé de façon récurrente de porter atteinte à l'image de l'encyclopédie libre (Moatti, 2015). Ces critiques mettaient en doute la fiabilité de son processus de construction des contenus, basé sur les internautes, quitte à intervenir directement sur les articles pour piéger le dispositif. Ainsi, dès 2007, Pierre Assouline fait introduire par ses étudiants une modification sur sa page le sacrant "champion de France du jeu de Paume ", qui lui servira ensuite dans un ouvrage dénonçant les failles de Wikipédia. Plus tard, en 2012, Jean-Noël Jeannerey, également membre du comité de rédaction de la revue L'Histoire, reprendra les mêmes arguments anti-wikipédiens. Moatti note que la plupart des critiques de Wikipédia émanent de milieux littéraires et indique «Leur prégnance en milieu littéraire, leur prolongement dans diverses controverses sur les méthodes éducatives, mais aussi leurs dérives occasionnelles vers la théorie du complot» (Moatti, 2015). Ainsi, parce que le modèle de Wikipédia a remis en question la distinction ordinaire entre savants et ignorants, en réalisant une forme d'expertise qui repose non pas sur la connaissance scientifique préalable mais sur l'expérience éditoriale au sein de la communauté (Willaime, 2015), l'encyclopédie

\footnotetext{
${ }^{14} \mathrm{https}: / /$ www.acpm.fr/Support/liberation

15 Jérôme Fenoglio, Louis Dreyfus, « 2017, une année de croissance pour le groupe Le Monde », Le Monde, 12 avril 2018.

${ }^{16}$ Alexandre Foatelli, «Les médias face à une crise de confiance généralisée », La Revue des Médias, 3 février 2017.

${ }^{17}$ Voir la page « Wikipédia:Réponses aux objections habituelles ».
} 
collaborative a été dénigrée dans les lieux de transmission du savoir légitime (Sahut, 2014). On retrouve dans certains médias un ton critique ${ }^{18}$, voire hostile, jusqu'en 2007, Norbert Bolz allant jusqu'à parler du « royaume des idiots » dans Der Spiegel ${ }^{19}$. Ces critiques se font plus rares par la suite car l'encyclopédie s'impose comme un acteur incontournable dans la diffusion des informations et renforce justement la présence des médias dans les sources de ses articles, médias qu'elle contribue ainsi à visibiliser.

Nous constatons que, dans le cas de Wikipédia comme dans celui des fake news, ces discours nourrissent un discrédit d'ordre général envers le rôle que peuvent tenir les internautes dans la production et la circulation de l'information. La représentation initiale du contributeur de Wikipédia dans la presse généraliste et celle actuelle, dans le cadre des fake news, de l'internaute partageant de l'information sur les réseaux sociaux numériques déclinent toutes deux les figures de l'ignorant ou du crédule, sujets à la manipulation voire vecteurs de celle-ci.

Les discours sur la vérité qui circulent dans l'espace public semblent ainsi en partie relever d'un certain opportunisme et des stratégies des acteurs visant à valoriser leur propre légitimité. Il en est de même concernant la capacité des internautes à participer à la circulation de l'information. Ces déplacements s'illustrent d'une autre façon dans la valorisation puis la mise au banc du journalisme participatif. En effet, après une période où plusieurs médias se sont montrés favorables au journalisme participatif ou citoyen, ouvrant même des blogs ou autres espaces dédiés sur leur propre site internet, la tendance suggère plutôt un (re)verrouillage de l'information. Or, la valorisation du journalisme participatif et citoyen par les médias s'inscrivait aussi dans des enjeux de légitimité envers leur public «En effet, la référence au peuple n'est souvent qu'un prétexte bien commode, un moyen de relégitimation d'une profession en crise » (Pélissier, Chaudy, 2009). Cet exemple illustre que les discours autour des publics et leur capacité informationnelle, supposée ou effective, s'intègrent dans des enjeux de reconnaissance des médias eux-mêmes (Tredan, 2007).

\section{Limites des modèles de prescription de l'information centralisés et verticaux}

Nous venons de noter que les discours de disqualification, dans le cas de Wikipédia ou concernant les fake news, sont issus d'experts historiques des domaines concernés qui remettent en cause des nouvelles formes de médiation de l'information ou des savoirs. Ils s'inscrivent dans des logiques fortement verticalisées dites «top-down», alors que ces médiations en réseaux s'organisent de façon beaucoup plus réticulaire. De par son mode de construction, le fonctionnement de ces modèles collaboratifs s'avère beaucoup plus difficile à contrôler, à cerner, et à rentabiliser commercialement.

Dans un entretien pour Wired en 2014, Tim Berners Lee indiquait "We need to redecentralise the Web" ${ }^{20}$. Et le co-inventeur du Web d'alerter sur les risques que feraient peser Facebook et Google notamment sur la vision fondatrice d'un Web libre et ouvert "The Web had failed instead of served humanity". Il ajoute qu'avec les géants du Web, le réseau s'est progressivement refermé et qu'il est maintenant sujet à tous les types de manipulations qu'il qualifie d' "anti-humains ». En France, plusieurs chercheurs en sciences de l'information et de la communication dénoncent régulièrement l'apparition d'enclosures, de mise en silos de l'information par les GAFA qui produisent des bulles closes, soumises à la toute puissance des algorithmes de classement (Cardon, 2015a) ${ }^{21}$. Inspirés par la théorie des communs, dont notamment les travaux d'Elinor Ostrom, ces chercheurs dénoncent une réappropriation de

\footnotetext{
${ }^{18}$ Daniel Schneiderman, «Wikipédia ses espoirs, ses menaces (pour les médias) », Libération, 14 octobre 2005.

${ }^{19}$ Norbert Bolz, «Le nouveau royaume des idiots?», Dossier La révolution Web 2.0 : Quand le Net devient une communauté, Courrier international (reprise de Der Spiegel), le 31 août 2006.

${ }^{20}$ Liat Clark, « We need to re-decentralise the Web », Wired, 6 février 2014.

${ }^{21} \mathrm{https}$ ://affordance.typepad.com/mon_weblog/2014/04/lutter-contre-les-enclosures-de-demain.html
} 
l'information par des acteurs économiques qui remettent en question le principe de neutralité du Net (Schafer, Le Crosnier, Musiani, 2011).

Le modèle de prescription, vers lequel semblent s'orienter les grands médias en s'associant avec Google, avance dans cette direction. Il s'agit d'un modèle pyramidal traditionnel du sachant vers le profane, mis sous tutelle car incapable de procéder par luimême à une évaluation des contenus qu'il consomme. Or, ce modèle s'oppose intrinsèquement au modèle collaboratif et réticulaire induit par la structure même d'internet. En effet, le réseau étant construit sur des technologies libres (TCP/IP, HTTP, HTML), les dynamiques collaboratives y sont depuis le départ encouragées, non seulement dans leur développement, à travers les communautés d'informaticiens partout sur la planète, mais aussi dans la façon dont les internautes échangent et produisent de l'information. A ce titre, le modèle d'organisation collaborative de Wikipédia, basé sur le Wiki, est un exemple indéniable de co-construction à grande échelle d'un édifice informationnel, sans qu'il soit nécessaire de centraliser les prises de décisions (Cardon, 2015b). Nous devons donc considérer qu'à la base même du succès d'internet et des réseaux sociaux, réside cette aspiration des internautes à vouloir échanger, produire, mais aussi évaluer par eux-mêmes les informations et les savoirs de façon collective.

Le renforcement de dispositifs verticalisés pose donc la question d'une forme de retour en arrière et de la pertinence de sa justification, au niveau discursif, par les " dérives » liées aux fake news. Nous constatons en effet que, depuis son lancement en février 2017, le Décodex est sujet à un double échec. D'abord un échec d'audience, comme le montrent les statistiques des tendances de recherche de Google ${ }^{22}$, avec des occurrences extrêmement faibles qui montrent le désintérêt global des internautes vis-à-vis de l'outil depuis son lancement pourtant très médiatisé. Mais aussi échec dans la constitution et l'évaluation du corpus médiatique traité par l'équipe du Décodex du Monde qui a changé les classifications utilisées pour catégoriser les sites d'information sans trouver de formule convaincante. Nos tests concernant huit sites spécialisés dans l'actualité liée à l'environnement ${ }^{23}$ montrent par exemple que le Décodex évalue peu de sites alternatifs d'information. Le Décodex tend à consacrer les médias classiques et, à l'opposé, à dénoncer des sites perçus comme politiquement extrêmes et dont les publications sont considérées par définition comme douteuses.

Nous pouvons ainsi constater que les fake news diffusées par certains médias traditionnels sont systématiquement considérées comme des erreurs alors que les mêmes fake news, relayées par des sites alternatifs, seront portées à leur discrédit. Ainsi, le site Révolution permanente est classé orange car il est accusé de relayer des informations inexactes mais l'exemple d'information inexacte qui est donné par le Décodex pour justifier ce choix avait été également relayé, et conforté avec des témoignages, par Reporterre ${ }^{24}$ et Marianne ${ }^{25}$ alors que ces deux médias sont classés comme fiables. Si Marianne s'inscrit dans la lignée des médias traditionnels, Reporterre pourrait être considéré comme un site d'information alternatif dans le sens où il transmet davantage que les autres médias les arguments et idées des militants environnementaux. Néanmoins, il est possible que son rédacteur en chef, issu du Monde, soit perçu comme une caution de fiabilité pour les journalistes du Décodex. Ainsi, une même information diffusée par des médias différents sera considérée par le Décodex comme plus ou moins à charge en fonction des énonciateurs respectifs.

\footnotetext{
$22 \mathrm{https} / /$ trends.google.fr/trends/explore?date=today $\% 205-\mathrm{y} \& \mathrm{geo}=\mathrm{FR} \& \mathrm{q}=\mathrm{d} \% \mathrm{C} 3 \% \mathrm{~A} 9 \operatorname{codex}$,Decodex

${ }^{23}$ Sur les huit sites testés dans le Décodex - reporterre.net; goodplanet.info ; novethic.fr ; femininbio.com ; wedemain.fr ; kaizen-magazine.com ; geo.fr ; larevolutiondestortues.fr - seul le plus connu, reporterre.net, est évalué.

${ }^{24}$ Camille Martin, "Trois témoins racontent comment un occupant de Tolbiac aurait été blessé à la tête », Reporterre, 21 avril 2018. Le titre de l'article a été modifié par Reporterre après l'éclaircissement de l'affaire.

25 https:/www.marianne.net/societe/tolbiac-aucun-blesse-grave-n-ete-hospitalise-reassure-la-police. L'article a été modifié.
} 
Dans ce contexte, on ne peut que s'interroger sur cette alliance de circonstance entre Google et les grands médias alors même qu'il y a quelques années, ce sont les médias et les agences de presse qui accusaient Google de piller leur contenu ${ }^{26}$. Certains étaient allés jusqu'à demander le déréférencement de leurs articles par le moteur de recherche. La dictature du clic, qui est inscrite au cœur du fonctionnement des algorithmes du géant de Mountain view, s'avère peu compatible avec une information de qualité nécessitant une démarche analytique des évènements et donc un rythme plus lent de publication. Le risque est donc que les médias accentuent leur course à l'audience, déjà effrénée, au détriment du traitement journalistique de fond des évènements. Et de perdre un peu plus leur indépendance en se plaçant sous les coupes de Google et de Facebook qui ne manqueront pas ensuite de leur indiquer comment procéder à leur manière s'ils veulent rester dans la course.

\section{Conclusion}

Cet article visait à mettre en exergue que la prolifération des discours et initiatives contre les fake news met en jeu de nombreux autres paramètres que le souci de vérité qu'expriment avec emphase certains médias traditionnels, nouveaux spécialistes du factchecking a posteriori ou partisans d'une standardisation a priori. Notre étude souligne que ces deux positions peuvent d'ailleurs également se recouper, comme c'est le cas pour l'AFP qui multiplie son implication dans les projets anti-fake news, notamment à travers Factuel, CrossCheck et Journalism Trust Initiative. En contrepoint de la dimension disruptive souvent prêtée au "phénomène » des fake news et qui, comme nous l'avons montré, sert à justifier des "solutions» permettant à certains acteurs de recentrer leur place dans le champ journalistique, nous voulions inscrire les fake news dans la continuité des discours de disqualification de ce qui paraît amateur et moins normé. Ainsi, dans les différents cas étudiés, ces discours proposent «des contrats relationnels permettant, implicitement ou explicitement, une sacralisation du représentant et une construction, fictionnelle et néanmoins généralisée, du représenté selon la figure du "profane" » (cf : appel à contribution).

Parmi les reconfigurations notables, la révision du jugement du Décodex à propos de Wikipédia, peu de temps après le lancement de celui-ci, semble significative. La typologie du classement du Décodex a été modifiée de sorte que Wikipédia est passée de la mise en garde initiale «Attention, ce site n'est pas une source à proprement parler ou sa fiabilité est trop variable pour entrer dans nos critères. Pour en savoir plus, cherchez d'autres sources et remontez à l'origine de l'information » à l'approbation générique pour tous les sites considérés comme fiables. Alors que la capacité de Wikipédia à garantir la véracité des informations publiées a souvent été mise en cause, les grandes plateformes d'information comme Facebook et Youtube ${ }^{27}$ se tournent vers elle pour lutter contre les fake news qu'ellesmêmes diffusent. Il semblerait qu'au sein du web participatif Wikipédia soit désormais placée dans un rôle inattendu de soutien à la vérité, témoignant ainsi d'un déplacement de la critique des pratiques collaboratives informationnelles de Wikipédia vers celles portant sur le partage d'informations sur les réseaux sociaux numériques, qu'il s'agirait maintenant d'encadrer. Si la revalorisation de Wikipédia par les médias peut aussi se fonder sur l'amélioration de sa fiabilité, ces médias semblent s'attacher à des pratiques surplombantes, coupées des lecteurs et verrouillant le champ de l'information, à l'opposé de son fonctionnement.

\footnotetext{
${ }^{26} \mathrm{https}$ ://www.huffingtonpost.fr/olivia-phelip/conflit-europe-presse-google b 2209662.html

${ }^{27}$ Dans le cadre de sa lutte contre les fake news, Youtube a ainsi récupéré les contenus des articles de Wikipédia pour les afficher sous les vidéos considérées comme étant à tendance complotiste. Will Oremus, « Fake news : YouTube sort du déni, mais est encore loin d »avoir résolu le problème ", Yann Champion (trad.), Slate, 23 mars 2018.
} 


\section{Références bibliographiques}

Alloing C., Vanderbiest N. (2018). « La Fabrique des rumeurs numériques. Comment la fausse information circule sur Twitter? », Le Temps des Médias, n³0, pp. 105-123.

Aristote (2014). Les Politiques, trad. Pellegrin P., Paris, Flammarion.

Badouard R. (2019). «Les controverses comme objet d'étude en SIC : le cas des "fake news" », Colloque Vers une nouvelle culture de la communication politique ?, Paris, 15 et 16 mai.

Bensaude-Vincent B. (2013). L'Opinion publique et la science, À chacun son ignorance, Paris, La Découverte.

Bigot L. (2018). " Rétablir la vérité via le fact-checking », Le Temps des Médias, n³0, pp. 62-76.

Bigot L. (2017). " Le fact-checking ou la réinvention d'une pratique de vérification », Communication \& langages, $\mathrm{n}^{\circ} 192$.

Cardon D. (2015a). À quoi rêvent les algorithmes : Nos vies à l'heure des big data, Paris, Seuil.

Cardon D. (2015b). « Surveiller sans punir : La gouvernance de Wikipédia », in BARBE Lionel, MERZEAU Louise, SCHAFER Valérie, (sous la direction de), Wikipédia, objet scientifique non identifié, Nanterre, Presses Universitaires de Paris X, pp. 15-39.

Cardon D. (2010). La Démocratie Internet. Promesses et limites, Paris, Seuil.

Jacobsson A., Jacobsson E.-M. (2010). «Quality of the News », The International Encyclopedia of Communication, First Edition.

Langlais P.-C. (2014). «La Négociation contre la démocratie: le cas Wikipédia », Négociations, $\mathrm{n}^{\circ} 21$, pp. 21-34.

Laugier S., Ogien A. (2017), Antidémocratie, Paris, La Découverte.

Moatti A. (2015). "Postures d'opposition à Wikipédia en milieu intellectuel en France », in Barbe L., Merzeau L., Schafer V., (sous la direction de), Wikipédia, objet scientifique non identifié, Nanterre, Presses Universitaires de Paris X, pp. 15-39.

Ouakrat A. (2019). « La qualité de l'information en ligne : entre transformation du champ et tentative de stabilisation normative », Journées de l'ANR PIL, Paris, 20 et 21 mai.

Pélissier N., Chaudy S. (2009). « Le journalisme participatif et citoyen sur Internet : un populisme dans l'air du temps ? », Quaderni.

Rancière J. (2004a). Aux Bords du politique, 1990, Gallimard.

Rancière J. (2004b), Le Maître ignorant, 1987, Paris, 10/18. 
Sahut G. (2014), « Les Jeunes, leurs enseignants et Wikipédia : représentations en tension autour d'un objet documentaire singulier », Documentaliste - Sciences de l'Information, 51 (2), pp. 70-79.

Sauvé M.-R. (2019). "Les fake news ("infausses") dans les médias québécois : perceptions des journalistes ", Colloque Fake news médium et médias au service d'imaginaires communautaires, Bordeaux, 15 et 16 avril.

Schafer V., Le Crosnier H., Musiani F. (2011). La neutralité de l'internet. : Un enjeu de communication, Paris, CNRS Editions, Les essentiels d'Hermès, 978-2-271-07265-8. 〈hal-

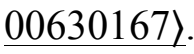

Tredan O. (2007). «Le "journalisme citoyen" en ligne : un public réifié ? », Hermès, La Revue.

Willaime P. (2015). "Une Analyse épistémologique de l'expertise dans Wikipédia », in Barbe L., Merzeau L., Schafer V., (sous la direction de), Wikipédia, objet scientifique non identifié, Nanterre, Presses Universitaires de Paris X, pp. 105-120.

\section{Sources journalistiques}

BOLZ Norbert, «Le nouveau royaume des idiots?», Dossier La révolution Web 2.0 : Quand le Net devient une communauté, Courrier international (reprise de Der Spiegel), 31 août 2006.

CLARK Liat, « We need to re-decentralise the Web », Wired, 6 février 2014.

COHEN Noam, « Conspiracy videos? Fake news? Enter Wikipedia, the 'good cop' of the Internet », Washington Post, 6 avril 2018.

FENOGLIO Jérôme et DREYFUS, Louis, « 2017, une année de croissance pour le groupe Le Monde », Le Monde, 12 avril 2018.

FOATELLI Alexandre, "Les médias face à une crise de confiance généralisée », La Revue des Médias, 3 février 2017.

MARTIN Camille, «Trois témoins racontent comment un occupant de Tolbiac aurait été blessé à la tête », Reporterre, 21 avril 2018.

MICHEL Patrick, " "Post-vérité" et "fake news" : fausses clartés et points aveugles », ACRIMED, 23 février 2018.

OREMUS Will, «Fake news: YouTube sort du déni, mais est encore loin d'avoir résolu le problème », CHAMPION Yann (trad.), Slate, 23 mars 2018.

PHÉLIP Olivia, "Presse contre Google, la guerre est déclarée... et les lecteurs oubliés !», HuffPost, 29 novembre 2012.

SCHERER Éric, « Contre la désinformation, Reporters sans frontières lance la Journalism Trust Initiative », Méta-Media, 3 avril 2018.

SCHNEIDERMAN Daniel, "Wikipédia ses espoirs, ses menaces (pour les médias)», Libération, 14 octobre 2005. 


\section{Sitographie}

Le HuffPost avec AFP, « Google va investir 300 millions de dollars pour lutter contre les fake news », HuffPost, 20 mars 2018.

Silicon, «Fake news : l’UE met la pression sur Google, Facebook et Twitter », 24 avril 2019.

Site de l'ACPM (Alliance pour les chiffres de la presse et des médias), page Libération, consulté le $1^{\text {er }}$ juin 2019.

Olivier Ertzscheid, "Lutter contre les enclosures de demain », blog Affordance.info, consulté le $1^{\text {er }}$ juin 2019

https://affordance.typepad.com/mon_weblog/2014/04/lutter-contre-les-enclosures-dedemain.html

Google Trends, mot clé "Decodex », consulté le 1" juin 2019

https://trends.google.fr/trends/explore?date=today $\% 205$ -

y\&geo $=$ FR\&q $=\mathrm{d} \% \mathrm{C} 3 \%$ A9codex,Decodex 